\title{
Efecto de la edad del macho cabrío en parámetros de calidad durante el proceso de criopreservación seminal
}

\author{
Age of male goat effect on quality parameters during the seminal cryopreservation process
}

\author{
Jorge Alonso Maldonado-Jáquez ${ }^{1 *}$, Lorenzo Danilo Granados-Rivera², \\ Pablo Alfredo Domínguez-Martínez ${ }^{3}$, Francisco Javier Pastor-López ${ }^{1}$, Leonardo Iván Vélez-Monroy ${ }^{1}$, \\ Uriel Figueroa-Viramontes ${ }^{1}$
}

\section{RESUMEN}

El objetivo de esta investigación fue evaluar el efecto de la edad de machos cabríos sobre parámetros de calidad seminal durante la criopreservación. Se utilizaron 33 machos asignados a tres grupos según la edad: adultos ( 31.4 meses); jóvenes (18.2 meses) y cabritos (8.5 meses). Los jóvenes y cabritos presentaron mayor número de eyaculaciones $(p<0.05)$ respecto a adultos. Volumen de eyaculado, motilidad y viabilidad del semen refrigerado, motilidad y viabilidad del semen congelado no fueron diferentes $(p>0.05)$ entre grupos. La motilidad del semen fresco con la viabilidad de semen fresco, concentración espermática, motilidad y viabilidad de semen refrigerado y edad se correlacionaron $(p<0.01)$. Asimismo, el diámetro testicular, edad y peso vivo tuvieron una alta correlación $(p<0.001)$ con la concentración espermática. Adultos y jóvenes tuvieron menor muerte de espermatozoides poscriopreservación. Se recomienda utilizar machos adultos o jóvenes para la conservación del semen a través de la criopreservación.

PALABRAS CLAVE

cabras locales, cruzamientos, inseminación, reproducción

\section{ABSTRACT}

The aim of this research was to evaluate the effect of buck age on semen quality parameters across the cryopreservation process. Thirty-three local males were divided into three groups: adult bucks (31.4 months old), young bucks (18.2 months old) and kid bucks $(8.5$ months old). Significant differences $(p<0.05)$ were found in number of ejaculations for young and kid bucks. No differences $(p>0.05)$ between groups were found for volume of ejaculate, motility and viability of refrigerated semen and motility and viability of refrigerated and frozen semen. Motility of fresh semen is correlated $(p<0.01)$ with motility and viability of fresh semen, sperm concentration, motility and viability of refrigerated semen and age. Testicular diameter, age and body weight have a high correlation $(p<0.001)$ with sperm concentration. Adult and young bucks presented less sperm death post-cryopreservation. It is recommended the use of adult or young bucks for seminal cryopreservation process.

KEYWORDS

local goats, breeding, insemination, reproduction

${ }^{1}$ Campo Experimental La Laguna, Instituto Nacional de Investigaciones Forestales, Agrícolas y Pecuarias. Matamoros, Coahuila, México.

${ }^{2}$ Campo Experimental General Terán, Instituto Nacional de Investigaciones Forestales, Agrícolas y Pecuarias. General Terán, Nuevo León, México.

${ }^{3}$ Campo Experimental Valle del Guadiana, Instituto Nacional de Investigaciones Forestales, Agrícolas y Pecuarias. Durango, Durango, México.

* Autor para correspondencia: Bulevar José Santos Valdez 1200, Centro. 27440 Matamoros, Coahuila, México.

Correo electrónico: maldonado.jorge@inifap.gob.mx 


\section{INTRODUCCIÓN}

En México, el sistema de producción caprino se lleva a cabo en las regiones áridas y semiáridas y donde una de las limitantes más marcadas está dada por la escasez de agua y forrajes en el periodo de sequía (Baraza et al. 2008). Asimismo, este sistema de producción es desarrollado por productores de escasos recursos económicos y dependen en alto grado del pastoreo en tierras comunales, en consecuencia, su productividad es baja (Escareño et al. 2011). A pesar de lo anterior, las cabras que se explotan en dicho sistema de producción representan una reserva genética valiosa debido a su adaptación fisiológica. Además, son una fuente económica importante para las familias de áreas rurales marginadas (González-Stagnaro 1993).

Por lo tanto, es necesario hacer investigación que ayude a que este sistema de producción incremente su productividad. Una opción, es el manejo reproductivo, el cual permite programar la época de partos, reducir los periodos improductivos, optimizar la prolificidad, así como aumentar la velocidad de mejora genética (Chemineau et al. 1993). Al respecto, en los programas de cruzamientos se han realizado ajustes, como el uso de reproductores a una edad temprana con el objetivo de reducir el intervalo generacional, además, de mejorar la taza de concepción y modificar la estacionalidad (Roca et al. 1991). Asimismo, se utilizan machos longevos (8 y 10 años), debido a que estos presentan un adecuado número espermático, con la desventaja de que en ellos se incrementa el número de espermatozoides con anormalidades (Akpa et al. 2013).

Para la Comarca Lagunera en el norte de México, los estudios de manejo reproductivo se han concentrado en la hembra y el manejo del fotoperiodo (Rincón et al. 2011; Bedos et al. 2016; Meza-Herrera et al. 2017), asimismo, los trabajos referentes a producción y calidad espermática no son muy numerosos (Ferreira y Flores 1993). Lo anterior es de gran relevancia, debido a que utilizar machos infértiles o peor aún, machos subfértiles en el empadre, afecta negativamente a todo el rebaño (Hoflack et al. 2006; Cardozo 2015), caso contrario de la hembra, donde el efecto negativo es individual. Por lo anterior, el objetivo del presente estudio fue evaluar el efecto de la edad del macho cabrío en los parámetros de calidad seminal durante el proceso de criopreservación.

\section{MATERIALES Y MÉTODOS}

Todos los métodos y el manejo de los machos cabríos utilizados en este estudio están estrictamente apegados a los lineamientos aceptados para el uso ético, cuidado y bienestar de los animales utilizados en investigación Internacional de acuerdo con la Federation of Animal Science Societies (FASS 2010), National Academy of Medicine (NAM 2002) e institucional, con la aprobación del proyecto titulado "Estrategia de investigación para el diagnóstico, documentación y conservación a largo plazo de los recursos genéticos del INIFAP (Ganado Criollo)".

El estudio se realizó en las instalaciones del módulo caprino y laboratorio de reproducción del Campo Experimental La Laguna, del Instituto Nacional de Investigaciones Forestales, Agrícolas y Pecuarias (Inifap) ubicado en Matamoros, Coahuila, México, dentro de la región conocida como Comarca Lagunera $\left(24^{\circ} 22^{\prime}\right.$ y $26^{\circ} 23^{\prime}$ latitud $N, 102^{\circ} 22^{\prime}$ y $104^{\circ} 47^{\prime}$ longitud $\mathrm{O}, 1100$ msnm). El clima corresponde a BWhw según la clasificación de Köpen modificado por García (1988), que se caracteriza por ser muy seco o desértico, semicálido con invierno fresco, y precipitación media anual de $240 \mathrm{~mm}$, la temperatura media anual a la sombra es de $25^{\circ} \mathrm{C}$, con rangos de $-1^{\circ} \mathrm{C}$ en invierno a $44^{\circ} \mathrm{C}$ en verano.

En el experimento se utilizaron 33 machos cabríos locales, mismos que fueron desarrollados bajo condiciones intensivas a partir del destete. Los machos fueron alojados en corrales grupales de $225 \mathrm{~m}^{2}$. Todos los animales fueron alimentados ad libitum con una dieta con base en heno de alfalfa, heno de avena y rye grass. Tuvieron acceso a agua fresca, limpia y a bloques de sales minerales en todo momento. Los animales fueron asignados a tres grupos de acuerdo con su edad. El grupo 1 (machos adultos; $n=11$ ) tiene edad promedio de 31.4 meses y peso vivo (PV) de 59.3 $\mathrm{kg}$; el grupo 2 (machos jóvenes; $\mathrm{n}=12$ ), de 18.2 meses de edad y $46.9 \mathrm{~kg}$ de PV y el grupo 3 (machos cabritos; $\mathrm{n}=10$ ), de edad promedio de 8.5 meses y $31.2 \mathrm{~kg}$ de PV.

El diámetro testicular en cada macho se midió en centímetros como la máxima dimensión alrededor del escroto en la porción media del testículo.

La colección de semen se realizó utilizando una vagina artificial. Se introdujo una hembra estrogenizada y un macho en un corral de $2 \times 2 \mathrm{~m}$. La vagina se preparó previamente con agua y aire a una temperatura de $38^{\circ} \mathrm{C}$ al momento de la colecta de semen. El semen se colectó cada día por 7 días, en los cuales los machos fueron expuestos en dos ocasiones a las hembras. El volumen del eyaculado se midió en tubos eppendorf que fueron utilizados para colectar la muestra. En cada etapa de la criopreservación (fresco, refrigeración y poscriopreservación) se determinó la motilidad (objetivo 4x) y viabilidad (objetivo 40x) del semen por observación directa en microscopio. 
La motilidad se contabilizó con al menos 200 espermatozoides, seleccionados al azar en un mínimo de cuatro campos del microscopio. La media de cuatro estimaciones sucesivas fue registrada como la motilidad final. La viabilidad fue evaluada en frotis teñidos con eosina-nigrosina. La concentración se midió con el equipo Spermacue modelo Photometer SDM (Delavan, Wisconsin, USA). Para la dilución del semen se utilizó un diluyente comercial (OptidylTM) con base de citrato y yema de huevo. El diluyente se agregó en dos etapas, al inicio de la refrigeración y 4 horas posteriores al término del periodo de estabilización. El empajillado se realizó por medio de succión.

La criopreservación se inició colocando pajillas a 10 cm del espejo del nitrógeno líquido por $15 \mathrm{~min}$, posteriormente se ubicó a $5 \mathrm{~cm}$ por $15 \mathrm{~min}$ y finalmente se sumergieron en nitrógeno líquido $\left(-196^{\circ} \mathrm{C}\right)$ y se almacenaron en un termo criogénico.

El análisis estadístico de los datos se realizó por medio de estadística no paramétrica, utilizando la prueba de Kruskall-Wallis con el test de comparación múltiple de Dunn. El nivel de significancia fijado para la totalidad de las pruebas fue de $p<0.05$. La determinación de relaciones se realizó con el análisis de correlación de Pearson con el programa InfoStat v.2008.

\section{Resultados}

Los machos jóvenes y cabritos eyacularon más veces $(p=0.03)$ respecto a machos adultos. En machos adultos y jóvenes hubo menos $(p=0.02)$ espermatozoides muertos post-criopreservación en comparación con los cabritos. No se encontraron diferencias $(p>0.05)$ entre grupos para el número de espermatozoides muertos después del proceso de refrigeración (figura $1)$. Los machos adultos en el semen fresco tuvieron mayor motilidad espermática $(p<0.05)$, viabilidad del semen y concentración espermática, así como mayor diámetro testicular (cuadro 1). No se encontraron diferencias entre grupos $(p>0.05)$ en las variables volumen del eyaculado, motilidad espermática del semen refrigerado, viabilidad del semen refrigerado, motilidad espermática del semen congelado y viabilidad del semen congelado.

Se encontró que la motilidad espermática del semen fresco se correlaciona $(p \leq 0.01)$ con viabilidad del semen, concentración espermática, motilidad espermática de semen refrigerado, viabilidad de semen refrigerado y edad (cuadro 2). Asimismo, la concentración espermática tuvo una correlación alta $(p<0.001)$ con el diámetro testicular, edad y PV. En

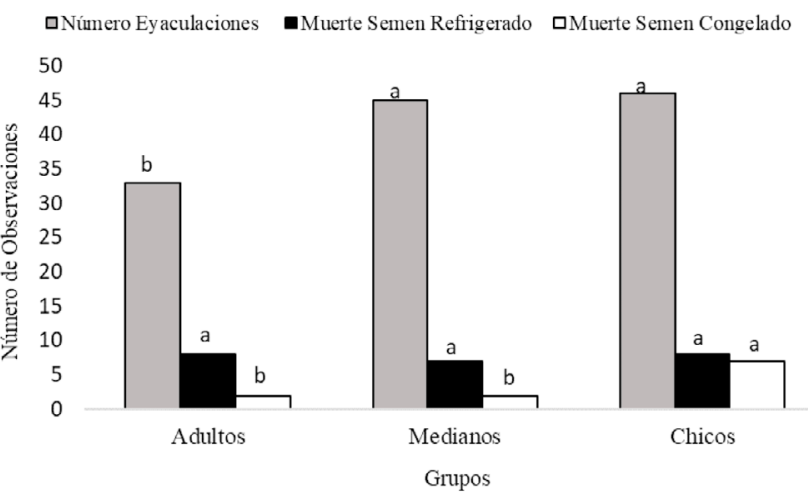

Figura 1. Número de eyaculaciones $y$ muerte de espermatozoides durante el proceso de refrigeración y poscriopreservación del eyaculado en machos cabríos locales de diferentes edades; diferente literal entre grupos indica significancia estadística $(p<0.05)$.

tanto, el diámetro testicular estuvo correlacionado $(p<0.001)$ con la edad y PV.

\section{Discusión}

La criopreservación de células espermáticas es un proceso complejo, ya que se incluye varias etapas críticas que pueden afectar los parámetros de calidad seminal (Küçük et al. 2014). Factores tales como época, edad del macho y prácticas de manejo afectan la calidad y la habilidad de congelación del semen (Gangwar et al. 2016). En este sentido, Swain et al. (2013) señalan que la variación estacional del año afecta la expresión de proteínas del plasma seminal, lo que está indirectamente relacionado con la calidad seminal. En este estudio, los machos se encontraban en el periodo de actividad sexual natural, por lo que la calidad seminal no estaría sujeta a una variación estacional. Por otra parte, Jiménez-Rabadán et al. (2012) indican efectos de manejo, por ejemplo, el método de colección, y señalan que el semen colectado por medio de una vagina artificial tiene mayor calidad. Con base en estos antecedentes se infiere que efectos externos no modificaron la calidad seminal de los machos cabríos y que las diferencias encontradas pueden ser atribuidas a las variables estudiadas.

El mayor diámetro testicular y concentración espermática de los machos adultos fue similar a los resultados reportados por Kerketta et al. (2014). Al respecto, el peso corporal y el diámetro escrotal aumentan con la edad (Lacuesta et al. 2015) debido a que todas las mediadas corporales en los animales tienen una influencia positiva con la edad (Akpa et al. 2013). En consecuencia, la edad es tomada por los 
Cuadro 1. Efecto de la edad de machos cabríos sobre características seminales durante el proceso de criopreservación.

\begin{tabular}{|c|c|c|c|c|c|c|c|c|c|c|}
\hline GRUPO & D.T. (cm) & Vol. (mL) & MotFre & ViaFre (\%) & Conc $\left(x 10^{9}\right)$ & MotRef & ViaRef (\%) & MotPc & & ViaPos (\%) \\
\hline Adultos & $\begin{array}{c}32.48 \pm \\
0.65^{\mathrm{A}}\end{array}$ & $0.74 \pm 0.06$ & $4.79 \pm 0.08^{\mathrm{A}}$ & $\begin{array}{c}88.48 \pm \\
1.05^{\mathrm{A}}\end{array}$ & $2.91 \pm 0.24^{\mathrm{A}}$ & $\begin{array}{c}3.16 \pm \\
0.13\end{array}$ & $66.4 \pm 2.03$ & $\begin{array}{l}2.43 \\
0.16\end{array}$ & \pm & $56.74 \pm 2.43$ \\
\hline Medianos & $\begin{array}{c}28.67 \pm \\
0.33^{\mathrm{B}}\end{array}$ & $0.7 \pm 0.06$ & $\begin{array}{c}4.6 \pm \\
0.08 \mathrm{~A}^{\mathrm{B}}\end{array}$ & $\begin{array}{c}85.8 \pm \\
2.27^{\mathrm{B}}\end{array}$ & $1.91 \pm 0.19^{\mathrm{B}}$ & $\begin{array}{c}3.22 \pm \\
0.13\end{array}$ & $68.16 \pm 1.69$ & $\begin{array}{l}3.18 \\
0.53\end{array}$ & \pm & $59.12 \pm 2.05$ \\
\hline Chicos & $\begin{array}{c}28.43 \pm \\
0.30^{\mathrm{B}}\end{array}$ & $0.6 \pm 0.06$ & $4.41 \pm 0.12^{\mathrm{B}}$ & $\begin{array}{c}81.65 \pm \\
2.37^{\mathrm{B}}\end{array}$ & $1.91 \pm 1.27^{\mathrm{B}}$ & $\begin{array}{c}2.88 \pm \\
0.12\end{array}$ & $63.42 \pm 1.41$ & $\begin{array}{l}2.39 \\
0.14\end{array}$ & \pm & $56.61 \pm 2.00$ \\
\hline$p$ Value & $<0.0001$ & 0.066 & 0.048 & 0.035 & 0.0025 & 0.076 & 0.15 & 0.21 & & 0.53 \\
\hline
\end{tabular}

Valores con literales diferentes son significativamente diferentes ( $p<0.05)$; valor \pm error estándar. Ad= Machos Adultos; Me= Machos Medianos; $\mathrm{Ch}=$ Machos chicos. D.T. = diámetro testicular; Vol = volumen de eyaculado; MotFre = motilidad espermática de semen fresco; ViaFre = viabilidad semen fresco; Conc = concentración espermática; MotRef= motilidad espermática de semen refrigerado; ViaRef = viabilidad semen refrigerado;

MotPos = motilidad espermática poscriopreservación; ViaPos = viabilidad poscriopreservación.

Cuadro 2. Matriz de correlaciones para características seminales en el proceso de congelamiento en machos cabríos locales.

\begin{tabular}{|c|c|c|c|c|c|c|c|c|c|c|c|}
\hline & Vol & MotFre & ViaFre & Conc & MotRef & ViaRef & MotPos & ViaPos & DT & Edad & Peso \\
\hline Vol & 1 & & & & & & & & & & \\
\hline MotFre & -0.13 & 1 & & & & & & & & & \\
\hline ViaFre & -0.04 & $0.49^{* * *}$ & 1 & & & & & & & & \\
\hline Conc & $0.71^{*}$ & $0.25^{* *}$ & 0.16 & 1 & & & & & & & \\
\hline MotRef & 0.06 & $0.39^{* * *}$ & 0.14 & 0.19 & 1 & & & & & & \\
\hline ViaRef & 0.09 & $0.34^{* * *}$ & 0.13 & 0.2 & $0.68^{*}$ & 1 & & & & & \\
\hline MotPos & $0.43^{* * *}$ & -0.15 & -0.0022 & -0.08 & 0.04 & 0.08 & 1 & & & & \\
\hline ViaPos & -0.1 & 0.16 & 0.17 & 0.04 & $0.23^{*}$ & $0.28^{* *}$ & $0.24^{*}$ & 1 & & & \\
\hline DT & 0.13 & 0.12 & 0.07 & $0.27^{* * *}$ & $0.08^{*}$ & 0.11 & -0.1 & 0.05 & 1 & & \\
\hline Edad & 0.14 & $0.24^{* *}$ & $0.18^{*}$ & $0.30^{* * *}$ & 0.14 & 0.12 & 0.02 & -0.01 & $0.5^{* * *}$ & 1 & \\
\hline Peso & 0.16 & 0.14 & 0.14 & $0.26^{* * *}$ & 0.11 & 0.07 & 0.15 & 0.01 & $0.52^{* * *}$ & $0.88^{*}$ & 1 \\
\hline
\end{tabular}

Valores con literales diferentes son significativamente diferentes ( $p<0.05)$; valor \pm error estándar. Ad= Machos Adultos; Me= Machos Medianos; $\mathrm{Ch}=$ Machos chicos. D.T. = diámetro testicular; $\mathrm{Vol}=$ volumen de eyaculado; MotFre = motilidad espermática de semen fresco; ViaFre = viabilidad semen fresco; Conc = concentración espermática; $\operatorname{MotRef}=$ motilidad espermática de semen refrigerado; ViaRef = viabilidad semen refrigerado;

MotPos = motilidad espermática poscriopreservación; ViaPos = viabilidad poscriopreservación.

caprinocultores como indicador para seleccionar a los sementales que utilizarán en el empadre, los cuales tienen que alcanzar una edad entre 17 y 19 meses (Ridler et al. 2012).

En el presente estudio la edad del macho cabrío no modificó el volumen de semen colectado, sin embargo, investigaciones reportan un efecto positivo de la edad en el volumen de semen eyaculado (Furstoss et al. 2009; Ramukhithi et al. 2011). Por otro lado, el tipo de alimentación que recibe el animal influye en la producción espermática (Oldham et al. 1978), y dado que los grupos de machos cabríos (adultos, jóvenes y cabritos) en estudio consumieron la misma dieta, no se esperaban cambios en el volumen de semen colectado.
La mayor calidad seminal de los machos adultos respecto a machos jóvenes y cabritos, encontrada en este estudio coincide con otras investigaciones (Al-Ghalban et al. 2004; Furstoss et al. 2009; Bitto y Egbunike 2012; Lacuesta et al. 2015). Lo anterior, es debido a que en machos adultos se ha completado el crecimiento y desarrollo del sistema reproductivo, caso contrario con machos en crecimiento (Ahsan et al. 2014). No obstante, en el presente estudio el número de eyaculados fue mayor en machos jóvenes y cabritos que en adultos, lo cual podría estar relacionada con la dieta que consumieron los machos en estudio, al respecto, Foote (1978), indica que una buena nutrición promueve un rápido crecimiento, actividad sexual temprana y la posibilidad de colectar semen a una edad temprana. 
No se encontraron reportes sobre correlaciones dentro del proceso de criopreservación, sin embargo, existe información que indica que todas las medidas testiculares de forma general muestran una fuerte correlación con la producción espermática (Almeida et al. 2007), asimismo, entre variables de calidad, por ejemplo, la motilidad espermática y la tasa de fertilidad (Hamidi et al. 2012). Por otra parte, se ha encontrado una fuerte correlación entre volumen y peso testicular y una correlación significativa entre la actividad de la arginasa en plasma seminal con ciertos parámetros espermatogénicos, que se pueden considerar como criterios para determinar la calidad seminal en machos cabríos (Agga et al. 2011; Türk et al. 2011).

\section{CONCLUSiones}

Se concluye que bajo las condiciones del presente estudio la calidad del semen en machos adultos y jóvenes fue mayor que en machos cabritos. Con base en ello, para la región lagunera, en México, se recomienda utilizar machos adultos ( $>30$ meses) o jóvenes $(>15$ meses) en los procesos de criopreservación o esquemas de cruzamiento para asegurar una buena calidad seminal durante los procesos de criopreservación. 


\section{LITERATURA CITADA}

Agga GE, Udala U, Regassa F, Wudie A. 2011. Body measurements of bucks of three goat breeds in Ethiopia and their correlation to breed, age and testicular measurements. Small Ruminant Research 95(2-3): 133-138. https://doi.org/10.1016/j.smallrumres.2010.09.011

Ahsan U, Kamran Z, Raza I, Ahmad S, Babar W, Riaz MH, Iqbal Z. 2014. Role of selenium in male reproduction-A review. Animal Reproduction Science 146(1-2): 55-62. https://doi.org/10.1016/j.anireprosci.2014.01.009

Akpa GN, Ambali AL, Suleiman IO. 2013. Body conformation, testicular and semen characteristics as influenced by age, hair type and body condition of Red Sokoto goat. New York Science Journal 6(7): 44-58.

Al-Ghalban AM, Tabba MJ, Kridli RT. 2004. Factors affecting semen characteristics and scrotal circumference in Damascus bucks. Small Ruminant Research 53(1-2): 141149. https://doi.org/10.1016/j.smallrumres.2003.10.003

Almeida AM, Schwalbach LMJ, Cardoso LA, Greyling JPC. 2007. Scrotal, testicular and semen characteristics of young Boer bucks fed winter veld hay: The effect of nutritional supplementation. Small Ruminant Research 73(1-2): 216-220. https://doi.org/10.1016/j.smallrumres.2007.02.001

Baraza E, Angeles R, García A, Valiente BA. 2008. Nuevos recursos naturales como complemento a la dieta de caprinos durante la época seca en el Valle de Tehuacán, México. Interciencia 33: 891-896.

Bedos M, Portillo W, Dubois J-P, Duarte G, Flores JA, Chemineau P, Keller M, Paredes RG, Delgadillo JA. 2016. A high level of male sexual activity is necessary for the activation of the medial preoptic area and the arcuate nucleus during the "male effect" in anestrous goats. Physiology and Behavior 165: 173-178.

Bitto II, Egbunike GN. 2012. The semen characteristics of pubertal West African Dwarf bucks. Pertanika Journal of Tropical Agriculture Science 35(2): 191-197.

Cardozo JA. 2015. La biología celular y molecular en la evaluación de la fertilidad del macho. Revista ACOVEZ 44(2): 17-19.

Chemineau P, Baril G, Delgadillo JA. 1993. Control hormonal de la reproducción del caprino. Revista Científica, FCV-LUZ 3(3): 197-210.

Escareño LM, Wurzinger M, Pastor F, Salinas H, Sölkner J, Iñiguez L. 2011. La cabra y los sistemas de producción caprina de los pequeños productores de la Comarca Lagunera, en el norte de México. Revista Chapingo Serie Ciencias Forestales y del Ambiente 17 (Edición Especial): 235-246.

[FASS] Federation of Animal Science Societies. [internet]. 2010. Guide for de care and use of agricultural animal an agricultural research and leaching. In Federation of Animal Science Societies. Acceso: 15 de agosto de 2016. Disponible en: http://www.fass.org/docs/agguide3rd/ Ag_Guide_3rd_ed.pdf

Ferreira NJ, Flores SM. 1993. El agua de coco (Cocus nucifera L) "in natura" integral y adicionada con citoquini- nas, como dilutor de semen caprino. Revista Científica, FCV-LUZ 3(3): 273-281.

Foote RH. 1978. Factors influencing the quantity and quality of semen harvested from bulls, rams, boars and stallions. Journal of Animal Science 47(Suppl. 2): 1-11.

Furstoss V, David I, Leboeuf B, Guillouet P, Boué P, Bodin L. 2009. Genetic and non-genetic parameters of several characteristics of production and semen quality in young bucks. Animal Reproduction Science 110(1-2): 25-36. https://doi.org/10.1016/j.anireprosci.2007.12.011

Gangwar C, Kharche SD, Kumar S, Jindal SK. 2016. Cryopreservation of goat semen: status and prospects. Indian Journal of Small Ruminants 22(1): 1-10. https://doi. org/10.5958/0973-9718.2016.00005.2

García E. 1988. Modificaciones al Sistema de Clasificación Climática de Köppen. Universidad Nacional Autónoma de México. D.F., México.

González-Stagnaro C. 1993. Comportamiento productivo de ovejas y cabras tropicales. Revista Científica, FCVLUZ 3(3): 173-196.

Hamidi A, Mamoei M, Mirzadeh Kh, Tabatebaei S, Roshanfekr H. 2012. Seasonal variations in semen characteristics in Arabic rams. Pakistan Veterinary Journal 32(1): 41-44.

Hoflack G, Van Soom A, Maes D, de Kruif A, Opsomer G, Duchateau L. 2006. Breeding soundness and libido examination of Belgian Blue and Holstein Friesian artificial insemination bulls in Belgium and The Netherlands. Theriogenology 66(2): 207-216. https://doi.org/10.1016/j. theriogenology.2005.11.003

Jiménez-Rabadán P, Ramón M, García-Álvarez O, Maroto-Morales A, del-Olmo E, Pérez-Guzmán MD, Bisbal A, Fernández-Santos MR, Garde JJ, Soler AJ. 2012. Effect of semen collection method (artificial vagina vs electroejaculation) extender and centrifugation on postthaw sperm quality of Blanca-Celtibérica buck ejaculates. Animal Reproduction Science 132(1-2): 88-95. https://doi.org/10.1016/j.anireprosci.2012.04.005

Kerketta S, Singh M, Patel BHM, Dutt T, Upadhyay D, Bharti PK, Sahu S, Kamal R. 2014. Relationship between age, body measurements, testicular measurements and total ejaculation of semen in local goat of Rohilkhand region. Small Ruminant Research 130: 193-196. https:// doi.org/10.1016/j.smallrumres.2015.07.006

Küçük N, Aksoy M, Uçan U, Ahmad E, Naseer Z, Ceylam A, Serin I. 2014. Comparison of two different cryopreservation protocols for freezing goat semen. Cryobiology 68(3): 327-331. https://doi.org/10.1016/j.cryobiol.2014.04.009

Lacuesta L, Orihuela A, Ungerfield R. 2015. Reproductive development of male goat kids reared with or without permanent contact with adult females until 10 months of age. Theriogenology 83(1): 139-143.

Meza-Herrera CA, Cano-Villegas O, Flores-Hernández A, Veliz-Deras FG, Calderón-Leyva G, Guillen-Muñoz JM, García C, Rosales-Nieto CA, Macias-Cruz U, Avenda- 
ño-Reyes L. 2017. Reproductive outcomes of anestrous goats supplemented with spineless Opuntia megacantha Salm-Dyck protein-enriched cladodes and exposed to the male effect. Tropical Animal Health Production 49: 1511-1516. https://doi.org/10.1007/s11250-017-1356-y

[NAM] National Academy of Medicine. 2002. Guide for the care and use of laboratory animals. Co-produced by the National Academy of Medicine-Mexico and the Association for assessment and accreditation of laboratory animal care international. D.F., Mexico.

Oldham CM, Adams NR, Gherardi PB, Lindsay DR, Mackintosh JB. 1978. The influence of level of feed intake on sperm-producing capacity of testicular tissue in the ram. Australian Journal of Agricultural Research 29(1): 173-179. https://doi.org/10.1071/AR9780173

Ramukhithi FV, Nedambale TL, Sutherland B, Lehloenya KC. 2011. Cryopreservation of South African indigenous goat semen. African Journal of Biotechnology 10(77): 17898-17902.

Ridler AL, Smith SL, West DM. 2012. Ram and buck management. Animal Reproduction Science 130(3-4): 180183. https://doi.org/10.1016/j.anireprosci.2012.01.012

Rincón RM, Aréchiga CF, Escobar FJ, Aguilera-Soto JI, Lopez-Carlos MA, Silva JM, Medina CA, Meza-Herrera CA, Valencia J. 2011. The male effect stimulus positively increased the ovarian and reproductive seasonality in Criollo Goats irrespective of a controlled photoperiodic regime. Journal of Applied Animal Research 39(3): 205211. https://doi.org/10.1080/09712119.2011.607720

Roca J, Martínez E, Vázquez JM, Ruíz S, Coy P. 1991. Influencia de la edad sobre los parámetros reproductivos de machos cabríos de raza Murciano- Granadina. Archivos de Zootecnia 40: 173-179.

Swain DK, Kumar T, Kumar A, Pandey V, Yadav S. 2013. Seasonal variations in seminal plasms protein profile of Barbari goats. Veterinary Practitioner 14(1): 171-172.

Türk G, Gür S, Kandemir FM. Sönmez M. 2011. Relationship between seminal plasma arginase activity and semen quality in Saanen bucks. Small Ruminant Research 97: 83-87. https://doi.org/10.1016/j.smallrumres.2011.01.015 